\title{
The Implementation of Serial Communication CSerial the Port Class
}

\author{
Naining Wen, Shangfu Gong, Yanjun Wang \\ College of Computer Science and Technology, Xi'an University of Science and Technology, Xi'an, 710054, China \\ E-mail: wnn03187@126.com
}

\begin{abstract}
Illustrating the advantages and disadvantages of several commonly used methods to develop serial communication program under Microsoft Windows, this article uses a serial communication class through a third party, namely the use the Remon Spekrijse provided free serial port class CSerial the Port class, complete the RS232 serial port communication between the examples show, CSerial Port is a very simple, convenient and convenient and simple multithreaded serial programming tool.
\end{abstract}

Keywords-tthe port CSerial class; serial communication; the microsoft windows

\section{INTRODUCTION}

The field of industrial control is often related to the problem of serial communication. Serial communication is a data communication technology of between the computer or computer and programmable control equipment. The serial communication with the use of a few lines, low cost, especially for remote transmission, to avoid multiple lines characteristic of inconsistency is widely used.. In the serial communications, requirement of communication both sides used a standard interface, so that different device can be conveniently connected for communication.

Different methods are adopted to achieve the data exchange between PC and MCU serial communication. Serial communication technology in such circumstances is particularly important. $\mathrm{VC}++6.0$ is an integrated development environment launched by Microsoft in 1998, with its powerful features, friendly interface, object-oriented programming and the flexibility of Active $\mathrm{X}$ and favored by the majority of software developers, being widely used in various fields. Among many ways of the application development of the $\mathrm{VC}++$ serial communication at present, the use of the $\mathrm{VC}++$ CSerial class, is an ideal one of the realization of serial communication method, as long as the understanding of a few member functions of this class, you can easily use it.

\section{COMPARISON OF SERIAL COMMUNICATION}

Comparison of the advantages and disadvantages of the development of several commonly used method of serial communication program.

The following methods listed are serial communication program developed based on Microsoft Windows ${ }^{[1]}{ }^{[2]}$ :

- Use the Windows API communication function;

- Use the Windows read and write port function _inp _inpw, _inpd _outpd or development driver directly to the serial port operation;
- Use third-party or write own communications;

- Use serial communications components, such as ActiveX control MSComn.

The first way, used widely among the above methods, has some difficulties in practical application due to its own complication. The second method need an understanding of the hardware circuit structure and theory, in-depth driver level, high degree of specialization. The third method uses the object-oriented technology package Win32 API functions, one for serial communication class, based on the understanding of several member functions of this class, it's easy to use. The fourth method is relatively simple, with a simple configuration of the serial port, but the only difficulty is puzzling VARIANT class, less the actual project.

In view of the above serial communication method of analysis, this paper uses a third method to provide underlying support for serial communication using the the Remon Spekrijse free serial port CSerial the Port class, and the class based on the complete RS232 interface serial communication protocol. The CSerial Port class is a very easy to use multi-threaded serial authoring tool, compared to MSComm control, packaged in this class do not need to add other files, and functions are open and transparent, allowing us to transform. CSerial Port class support line (Non MODEM) to connect the serial programming operation, programs written in Windows2000/XP operating system can function well..

\section{MAIN FUNCTION}

CSerial Port workflow and frequently use several main functions

CSerial Port class is based on multi-threaded, so the workflow is as follows: when it comes to receiving data, the serial port parameters are set up, and then the serial port monitoring thread open, serial port monitoring thread to monitor the serial received data flow, control events or other serial event, and news way to notify the main program to stimulate message processing function to the data processing; send data can be sent directly to the serial port.

Several key functions are frequently used for communication CSerial the Port class[3]:

- Serial port initialization function InitPort(): This function is used to initialize serial port, set the serial communications parameters including need to open the serial port, baud rate, parity mode, data bits, stop bits.but each time only a serial pot initialization.

- To start serial communication monitor thread function StartMonitoring(): serial port initialization is successful, you can call the BOOL 
StartMonitoring() to start the serial port monitoring thread. Thread starts successfully return TRUE; to call InitPort() and StartMonitoring(), serial port is opened, a variety of serial port status and events can be monitored.

- $\quad$ Serial communication thread function (): the thread function will work as the thread is started, once a thread is activated, it will be responsible for monitoring the serial port to receive the string event, send string event and closed string event, and the event scheduling and related processing function, then completing the serial port operation.

- Pause or stop monitor thread function StopMonitoring(): this function to pause or stop serial monitoring, and call the function, the serial ports is still occupied.

- Close serial function ClosePort(): the function is to close the serial port, the release of the serial ports.

- Through the serial port to send/write serial function WriteToPort(): this function is completed serial port to send data to write the serial port function.

- Message processing function WM_COMM_ RXCHAR(): this function is used for receiving the serial received character.

\section{EXAMPLE ANALYSES}

In computer peripherals, the RS-232 serial port programming simple, convenient control, therefore in the laboratory and industrial applications of the various control systems, the serial port is used with external computer control system between the data transmission channel. According to the different conditions can be realized on the serial programming flexibility. RS-232 symbols and functions as shown in Table 1

TABLE I. RS-232 SYMBOLS AND FUNCTIONS

\begin{tabular}{|l|l|}
\hline Symbol & Function \\
\hline TXD & Transmit data \\
\hline RXD & Receive data \\
\hline RTS & Request to send \\
\hline CTS & Clear to send \\
\hline DSR & Data set ready \\
\hline GND & Signal ground \\
\hline DCD & Data signal detection \\
\hline DTR & Data terminal ready \\
\hline RI & Ring indicator \\
\hline
\end{tabular}

Using the CSerial Port class in a configuration where the two serial the same computer RS232 interface between the serial communication protocol testing, on the COM1 data information is transmitted to the COM2, the data information includes seven sensors (T1 T5) work status, seven kinds of equipment (main bureau fan, deputy bureau fan, fore-field, inverter, main power source, auxiliary power source, higher power) working state transmission. The following from the build debugging environment, user communication protocol for the preparation of, communication data packet processing method and program testing from four aspects.

\section{A. Hardware debugging environment}

As shown in Figure 1, the hardware debugging environment is a serial port connection, Using DB9 pin plugs to connect, 2 serial ports COM1 and COM2 of the PC to connect the data cable, making between COM1 and COM2 can communicate [4].

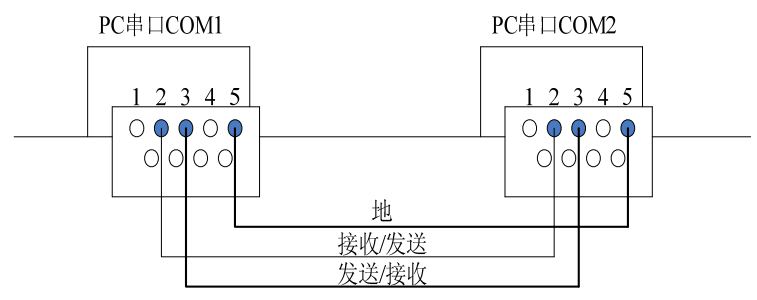

Figure 1. RS-232 serial connection schematic

\section{B. The preparation of user communication protocol}

User layer of the preparation of serial port communication protocol is very arbitrary, but there are several principles that must be followed[5].

- A header is needed in data packet.

- A packet fail must be stored in non-fixed-length data packet.

- Fixed-length packet specified length, for variable length packets, each packet in the specified location description.

- Data must be checked.

- New line symbol must be added in the end.

- Be as short as possible, in order to facilitate the updating of the data. Based on the above principle, this paper makes a discussion of a communication protocol.

In this paper, using the defined communication protocol, the protocol consists of three bytes, the double bureau fans system with seven sensors (T1 T5) working condition, seven kinds of equipment (main bureau fan, deputy bureau fan, fore-field, inverter, main power source, auxiliary power source, higher power) working state transmission. The first byte is headed byte, the most significant bit (seventh) is 1 , the highest bit of the other two bytes is 0 , so that the recipient as long as the judgment of the highest bit of each byte to know of a packet byte, third byte parity bit high, and the remaining seven is the XOR value of the top two, the following specific communication protocol.

First bytes: MSB (seventh) is 1, said first byte, other bytes in the bit to 0 ; zero to sixth said seven sensor (T1 T5), 1 said alarm, 0 said to work properly.

Second bytes: seventh is 0 , indicating non-first byte. The rest of you for seven kinds of equipment working state, 1 normal, 0 means stop.

Third bytes: the byte instruction checksum byte, seven to 0 , said the non-first byte. After seven-byte is the first two 
bytes of non-highest bit of the first two bytes (0 6) that the number of "XOR" of the results individually, as far as to determine the command transmission is correct.

In summary, the packet communication protocol: \$(1)(2)

(3) (4)(5)(6) (8)(9)(10)(11)(12)(13)(14)*hh $<\mathrm{CR}><\mathrm{LF}>$

In which:

\$: String head, the first character;

(1)(2)(3)(4)(6): control seven sensor status;

(8)(9)(10)(11)(12)(13)(14): control 7 device status;

*: An asterisk, the end of the string;

$<\mathrm{CR}>$ : Carriage return-control characters;

$<$ LF $>$ : Newline control characters.

\section{Communication data packet processing method}

The processing of the packet side while receiving treatment, each character received to judgment. Whenever the serial port buffer in program design, serial communication events is triggered when one or more characters, the event-driven serial communication handler, the received packet processing.

\section{Program flow diagram}

Computer program flow as shown in the figure 2 . After the computer starts, firstly the serial port is initialized, then, begins to enter data, sends or receives data. if there is no end, continue to receive or send data. If after the processing is completed, then wait for the next data [6].

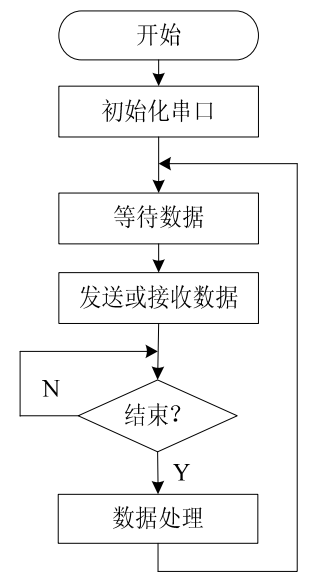

Figure 2. Computer program flow

\section{E. Program test}

Having connected the serial cable, we need test two serial communication protocol between the tested program interface, as shown in Figure 3, according to the actual connection, in accordance with the serial 1 in COM1, serial 2 in COM2, respectively, to open serial port, then the check box is selected on the sensor T5, main power source, auxiliary power source, higher power, deputy bureau fan, fore-field on the check box is selected, click the "Send" button, below the corresponding indicator light turns red, in Figure 3. each edit box is able to receive and send information, the programming was successful. To complete the task transmitted from the data on the COM1 to COM2.

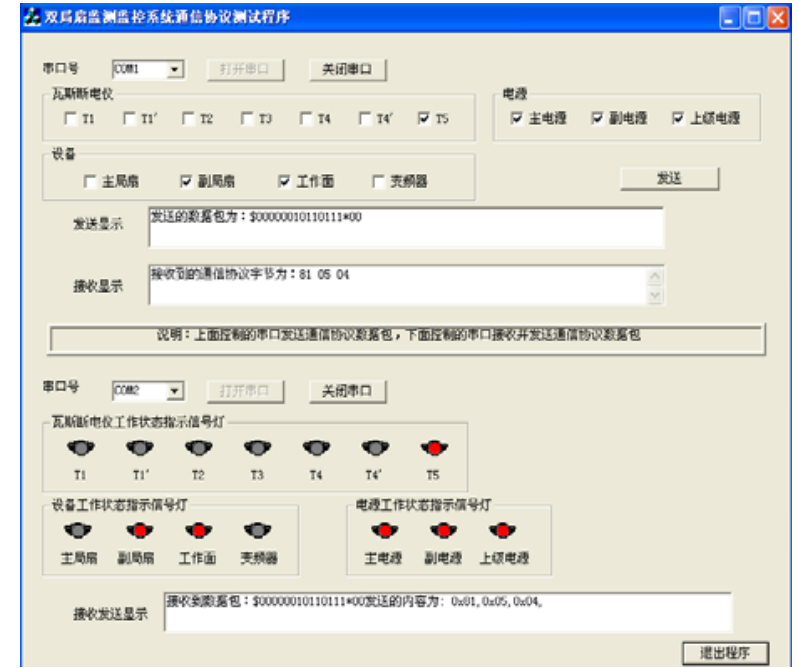

Figure 3. Sensors T5 exceeds the test the communication protocol interface

\section{CONCLUSIONS}

In this paper, a very proper multi-threaded serial writing tool CSerial Port class is presented, and the class based on the completion of a RS232 interface serial communication protocol, At the same time can obtain the practical and effective serial number, and can realize the single thread query receiving data and multi thread monitor receiving data; wherein the single thread query timeout function precision reach microsecond level; and the serial control receiving function in high speed transceiver when the phenomenon of missing data are improved[7]. By using CSerial Port class, it is possible to complete the general serial programming tasks within a very short time you can build a good serial communication framework, so that programmers will be doing well in the framework, and focus on the preparation of the communication protocol and data processing.

\section{REFERENCES}

[1] X.Y. LI, Visual C++ Serial Communication Technology and Engineering Practice. Beijing. People's Posts and Telecommunications Press, 2001.

[2] D. Chapman, Learning to Use the Visual $\mathrm{C}++6.0$. Beijing, Tsinghua University Press, 1999.5.

[3] S.L. TAN, Visual C++ Serial Communication Engineering Development Instance of the Navigation. Beijing: People's Posts \& Telecom Press, 2003.7.

[4] J.Y. YANG. "Based on the VisualBasic and RS232 serial communication temperature monitoring system". electronic test, vol12, pp.47-50, 2007.

[5] J.W. GONG, Visual C++ / Turbo C Serial Communication Programming Practice. Beijing, Publishing House of Electronics Industry, 2004.

[6] Y.R. MAO, "realization of serial communication between microcomputers and computer”. instrumentation analysis monitoring, vo01, pp.45-48, 2007.

[7] B.Y. SONG, J.H. WU, and B. HUANG, "Research on Highly Efficient Serial Communication in Microcontroller Unit," technology review, vol1, pp.27-29, 2010. 